\title{
ON THE TOPOLOGICAL STRUCTURE OF THE SOLUTION SET FOR A SEMILINEAR FUNCTIONAL-DIFFERENTIAL INCLUSION IN A BANACH SPACE
}

\author{
GIUSEPPE CONTI \\ Istituto di Matematica Applicata, Facoltà di Architettura \\ Università di Firenze, Italy \\ VALERI OBUKHOVSKII \\ Department of Physics and Mathematics \\ Voronezh Pedagogical University, Russia \\ PIETRO ZECCA \\ Dipartimento di Sistemi e Informatica, Università di Firenze \\ via S. Marta 3, 50139 Firenze, Italy \\ E-mail: pzecca@ingfi1.ing.unifi.it
}

Abstract. In this paper we show that the set of all mild solutions of the Cauchy problem for a functional-differential inclusion in a separable Banach space $E$ of the form

$$
x^{\prime}(t) \in A(t) x(t)+F\left(t, x_{t}\right)
$$

is an $R_{\delta}$-set. Here $\{A(t)\}$ is a family of linear operators and $F$ is a Carathéodory type multifunction. We use the existence result proved by V. V. Obukhovskiü [22] and extend theorems on the structure of solutions sets obtained by N. S. Papageorgiou [23] and Ya. I. Umanskiǔ [32].

Introduction. Beginning in the seventies the multivalued Cauchy problem in abstract spaces has been studied by many authors; we mention the existence theorems obtained by Chow and Schuur [6], Muhsinov [20], De Blasi [8], Anichini and Zecca [3], Sentis [26], Pavel and Vrabie [25], Tostonogov [27] and [28] and Kisielewicz [16]. The first approach to the structure of the solution set was by Davy [7] in the finite dimensional case. He proved that the set $S$ of solution is a continuum in $C\left([0, T], \mathbb{R}^{n}\right)$. Later Lasry and Robert [18] showed that $S$ is acyclic whenever $F$ has compact and convex values and is Hausdorff

1991 Mathematics Subject Classification: Primary 34A60; Secondary 34K30, 93B52, 93C20.

The paper is in final form and no version of it will be published elsewhere. 
upper semicontinuous (u.s.c). Subsequently Himmelberg and Van Vleck [13] proved that $S$ is a compact $R_{\delta}$ when $F$ is (u.s.c.) and bounded. De Blasi and Myjak [9] extended this result to the case where $F$ is Carathéodory. Only recently has the analogous problem been studied in abstract spaces. We note that in these studies the method of proof usually tries to follow the technique for the result in finite dimensions, adding hypotheses which guarantee the existence of solutions. In this way in 1982 Tostonogov first (see [29] and [30]) generalized a theory of Davy, and later Papageorgiou [24] extended to separable Banach spaces a result of Himmelberg and Van Vleck.

In this paper we show that the set of all mild solutions of the Cauchy problem for a functional-differential inclusion in a separable Banach space $E$, of the form

$$
x^{\prime}(t) \in A(t) x(t)+F\left(t, x_{t}\right),
$$

where $\{A(t)\}$ is a family of linear operators and $F$ is a Carathéodory type multifunction is a $R_{\delta}$-set. In order to achieve the result we use an existence theorem due to Obukhovskiu ([22]) and we extend theorems on the structure of the solution sets obtained by Papageorgiou [23] and Umanskiı [32]. For other results on the structure of solution sets we refer to the forthcoming survey of Dragoni, Macki, Nistri and Zecca [11].

1. Preliminaries. Let $V$ and $W$ be topological spaces; a multivalued map (multimap) $\mathcal{F}: V \multimap W$ is said to be:

(a) upper semicontinuous (u.s.c.) if $\mathcal{F}^{-1}(U)=\{x \in V \mid \mathcal{F}(x) \subset U\}$ is an open subset of $V$ for every open $U \subseteq W$;

(b) continuous if $\mathcal{F}$ is u.s.c. and lower semicontinuous, i.e. $\mathcal{F}^{-1}(D)$ is a closed subset of $V$ for every closed $D \subseteq W$.

Let us note (see, for example [4]) that if an u.s.c. multimap $\mathcal{F}: V \multimap W$ has compact values then the image $\mathcal{F}(K)$ of any compact set $K \subset V$ is relatively compact.

Let $\mathcal{E}$ be an infinite-dimensional Banach space. A function $\beta: 2^{\mathcal{E}} \rightarrow \mathbb{R}_{+}$is said to be a measure of noncompactness in $\mathcal{E}$ if

$$
\beta(\overline{\operatorname{co}} \Omega)=\beta(\Omega)
$$

for every $\Omega \in 2^{\mathcal{E}}$, where $\overline{c o}$ denotes the closure of the convex hull of the set (see, for example, [1]).

The measure of noncompactness $\beta$ is said to be:

(a) monotone if $\Omega_{1} \subseteq \Omega_{2}$ implies $\beta\left(\Omega_{1}\right) \leq \beta\left(\Omega_{2}\right)$;

(b) algebraically semiadditive if $\beta\left(\Omega_{1}+\Omega_{2}\right) \leq \beta\left(\Omega_{1}\right)+\beta\left(\Omega_{2}\right)$ for all $\Omega_{1}, \Omega_{2} \in 2^{\mathcal{E}}$;

(c) nonsingular if $\beta(\Omega \cup\{a\})=\beta(\Omega)$ for all $\Omega \in 2^{\mathcal{E}}, a \in \mathcal{E}$;

(d) regular if $\beta(\Omega)=0$ is equivalent to the relative compactness of $\Omega$.

One of the well-known examples of the measure of noncompactness possessing the properties (a)-(d) is the Hausdorff measure of noncompactness

$$
\chi(\Omega)=\inf \{\varepsilon>0 \mid \Omega \text { has a finite } \varepsilon \text {-net }\} .
$$

In what follows $E$ will denote a separable Banach space.

Let us note the following properties of $\chi$. 
LEMma 1.1. If $B$ is a bounded linear operator in $E$ then

$$
\chi(B D) \leq\|B\| \chi(D)
$$

for any bounded $D \subset E$.

Lemma 1.2. If $\Omega \subseteq C([a, b] ; E)$ is bounded and equicontinuous then

$$
\varphi(\Omega):=\sup _{t \in[a, b]} \chi(\Omega(t))=\chi_{c}(\Omega)
$$

where $\Omega(t)=\{y(t) \mid y \in \Omega\}$ and $\chi_{c}$ is the Hausdorff measure of noncompactness in $C([a, b] ; E)$.

The following statement expressing the continuity property of the measure of noncompactness can be easily verified.

LEMMA 1.3. Let $\beta$ be a monotone, nonsingular and regular measure of noncompactness in $\mathcal{E}$ and $\left\{\mathcal{K}_{n}\right\}, n=1,2, \ldots$ is the sequence of nonempty closed subsets of $\mathcal{E}$ such that $\mathcal{K}_{n+1} \subseteq \mathcal{K}_{n}, n=1,2, \ldots$ and $\beta\left(\mathcal{K}_{n}\right) \rightarrow 0$ while $n \rightarrow \infty$. Then

$$
\mathcal{K}=\bigcap_{n \geq 1} \mathcal{K}_{n}
$$

is a nonempty compact set.

A multifunction $G:[a, b] \multimap E$ with compact values is said to be measurable if it satisfies any of the following two equivalent conditions:

(i) the set $G^{-1}(U)$ is measurable for every open $U \subseteq E$;

(ii) there exists the sequence $\left\{g_{n}\right\}_{n=1}^{\infty}$ of measurable functions $g_{n}:[a, b] \rightarrow E$ such that $G(t)=\overline{\left\{g_{n}(t)\right\}_{n=1}^{\infty}}$ for all $t \in[a, b]$ (see, for example, [5]).

By the symbol $S_{G}^{1}$ we will denote the set of all Bochner integrable selectors of the multifunction $G:[a, b] \multimap E$, i.e.

$$
S_{G}^{1}=\left\{g \in L^{1}([a, b], E) \mid g(t) \in G(t) \text { a.e. }\right\} .
$$

If $S_{G}^{1} \neq \emptyset$ then the multifunction $G$ is called integrable and

$$
\int_{\mathcal{I}} G(s) d s:=\left\{\int_{\mathcal{I}} g(s) d s \mid g \in S_{g}^{1}\right\}
$$

for every measurable set $\mathcal{I} \subseteq[a, b]$. Clearly if $G$ is measurable and integrably bounded (i.e. there exists $\alpha \in L_{+}^{1}([a, b])$ such that $\|G(t)\|:=\max \{\|y\| \mid y \in G(t)\} \leq \alpha(t)$ a.e.) then $G$ is integrable.

We will need also the following property (see [22]).

Lemma 1.4. Let the multifunction $G:[a, b] \multimap E$ with bounded values be integrable, integrably bounded and

$$
\chi(G(t)) \leq \gamma(t) \quad \text { a.e. on }[a, b],
$$

where $\gamma \in L_{+}^{1}([a, b])$. Then

$$
\chi\left(\int_{\mathcal{I}} G(s) d s\right) \leq \int_{\mathcal{I}} \gamma(s) d s
$$


for every measurable set $\mathcal{I} \subseteq[a, b]$. In particular, if $G$ is measurable and integrably bounded then

$$
\chi(G(\cdot)) \in L_{+}^{1}([a, b])
$$

and

$$
\chi\left(\int_{\mathcal{I}} G(s) d s\right) \leq \int_{\mathcal{I}} \chi(G(s)) d s .
$$

At last, recall that a metric space is an $R_{\delta}$-set if it may be represented as an intersection of a decreasing sequence of compact contractible sets (see [14]). Every $R_{\delta}$-set is acyclic. For the definition of acyclic set see e.g. [21].

2. Results. Let $E$ be a separable Banach space; for $T>0, \tau>0$ let us denote $\mathcal{D}=C([-\tau, T] ; E), \mathcal{C}=C([-\tau, 0] ; E)$. For $x \in \mathcal{D}$ and $t \in[0, T]$ the function $x_{t} \in \mathcal{C}$ is defined by the relation $x_{t}(\theta)=x(t+\theta)$.

We will consider the Cauchy problem for the functional-differential inclusion of the form

$$
\begin{aligned}
x^{\prime}(t) & \in A(t) x(t)+F\left(t, x_{t}\right), & & t \in[0, T] \\
x(t) & =x_{0}(t), & t & \in[-\tau, 0]
\end{aligned}
$$

for a given initial function $x_{0} \in \mathcal{C}$ under the following assumptions.

(A) $\{A(t)\}_{t \in[0, T]}$ is a family of closed linear, not necessarily bouneded operators in $E$ generating a strongly continuous evolution operator $K: \Delta \rightarrow \mathcal{L}(E)$ where $\Delta=\{(t, s) \in[0, T] \times[0, T] \mid 0 \leq s \leq t \leq T\}$ and $\mathcal{L}(E)$ is a space of all bounded linear operators in $E$ (see [17], [19]). We will suppose also that $K$ is continuous with respect to the norm of the space $\mathcal{L}(E)$ while $s<t$.

Let $K v(E)$ denote the collection of all nonempty compact convex subsets of $E$.

We will suppose that the multimap $F:[0, T] \times \mathcal{C} \rightarrow K v(E)$ satisfies the following conditions:

(F1) for every $c \in \mathcal{C}$ the multifunction $F(\cdot, c):[0, T] \rightarrow K v(E)$ has a measurable selection;

(F2) for a.e. $t \in[0, T]$ the multimap $F(t, \cdot): \mathcal{C} \rightarrow K v(E)$ is u.s.c.;

(F3) $\|F(t, c)\| \leq \alpha(t)+\beta(t)\|c\|_{\mathcal{C}}$ for every $c \in \mathcal{C}$ and a.e. $t \in[0, T]$ where $\alpha, \beta \in$ $L_{+}^{1}([0, T])$

(F4) for every nonempty bounded equicontinuous set $D \subset \mathcal{C}$ we have

$$
\chi(F(t, D)) \leq k(t) \varphi(D) \quad \text { a.e. } t \in[0, T]
$$

where $\chi$ is the Hausdorff measure of noncompactness in $E ; \varphi$ is the measure of noncompactness in $\mathcal{C}$ defined in Lemma 1.2 and $k \in L_{+}^{1}([0, T])$.

It is clear that condition (F1) is fulfilled if the multifunction $F(\cdot, c)$ is measurable for every $c \in \mathcal{C}$. For $x \in D$ let us denote by $H_{x}$ the multifunction $H_{x}:[0, T] \rightarrow K v(E)$ given by $H_{x}(t)=F\left(t, x_{t}\right)$. From conditions (F1)-(F3) it follows that $S_{H_{x}}^{1} \neq \emptyset$ for every $x \in D$ (see, for example, [30]). 
Definition 2.1. A function $x \in \mathcal{D}$ is said to be a mild solution of the problem (1)-(2) provided

$$
\begin{array}{ll}
x(t)=x_{0}(t), & t \in[-\tau, 0], \\
x(t)=K(t, 0) x(0)+\int_{0}^{t} K(t, s) f(s) d s, & t \in[0, T],
\end{array}
$$

where $f \in S_{H_{x}}^{1}$.

The following existence theorem was proved by V. V. Obukhovskiu [22].

THEOREM 2.1. Under assumptions (A), (F1)-(F4) the set $\Sigma_{x_{0}}^{F}$ of all mild solutions of the problem (1), (2) is nonempty and compact in $\mathcal{D}$.

The main result of this paper is the following theorem describing the topological structure of the set $\Sigma_{x_{0}}^{F}$.

THEOREM 2.2. Under assumptions $(\mathrm{A}),(\mathrm{F} 1)-(\mathrm{F} 4)$ the set $\Sigma_{x_{0}}^{F}$ is an $R_{\delta}$-subset of $\mathcal{D}$.

To prove this fact we need a few preliminaries. First of all, let us note that we may assume, without loss of generality, that $F$ satisfies the following estimation:

(F3') $\|F(t, c)\| \leq \gamma(t)$ for every $c \in \mathcal{C}$ and a.e. $t \in[0, T]$ where $\gamma \in L_{+}^{1}([0, T])$.

In fact, let $\left\|\Sigma_{x_{0}}^{F}\right\|_{\mathcal{D}} \leq M ; B(0, M)$ be a closed ball in the space $\mathcal{C}$ and $\rho: \mathcal{C} \rightarrow$ $B(0 ; M)$ be a radial retraction. Then it is easy to see that the multimap $\widehat{F}:[0, T] \times \mathcal{C} \rightarrow$ $K v(E)$, defined by $\widehat{F}(t, c)=F(t, \rho c)$, satisfies conditions (F1), (F2), (F4) (note that $\rho$ is a Lipschitz map), the condition (F3') with $\gamma(t)=\alpha(t)+\beta(t) M$ and the set $\Sigma_{x_{0}}^{F}$ coincides with the set of all mild solutions of the problem

$$
\begin{aligned}
x^{\prime}(t) & \in A(t) x(t)+\widehat{F}\left(t, x_{t}\right), & & t \in[0, T] ; \\
x(t) & =x_{0}(t), & & t \in[-\tau, 0] .
\end{aligned}
$$

Therefore in what follows we will suppose that the multimap $F:[0, T] \times \mathcal{C} \rightarrow K v(E)$ satisfies the conditions (F1), (F2), (F4) and (F3') instead of (F3).

Now let us prove the following proposition.

LEMMA 2.1. Under assumptions (A), (F1)-(F4) there exists a nonempty compact convex subset $X \subset \mathcal{D}$ such that:

(i) $x(t)=x_{0}(t), t \in[-\tau, 0]$ for all $x \in X$;

(ii) $K(t, 0) x_{0}(0)+\int_{0}^{t} K(t, s) \overline{\operatorname{co}} F\left(s, X_{s}\right) d s \subseteq X(t), t \in[0, T]$ where $X_{s}=\left\{x_{s} \mid x \in\right.$ $X\} \subset \mathcal{C}$.

P r o of. Let us construct the decreasing sequence of closed convex sets $\left\{X^{i}\right\}_{i=1}^{\infty} \subset \mathcal{D}$ by the following inductive process.

Let $\mathcal{N} \subset \mathcal{D}, \mathcal{N}=\left\{x \in \mathcal{D} \mid x(t)=x_{0}(t), t \in[-\tau, 0]\right\}$ and

$$
X^{0}=\left\{x \in \mathcal{N} \mid\|x(t)\| \leq M_{1}, t \in[0, T]\right\}
$$

where $M_{1}=r\left(\left\|x_{0}(0)\right\|+\int_{0}^{T} \gamma(s) d s\right), r=\max _{(t, s) \in \Delta}\|K(t, s)\|$. 
Then $X^{n}=\overline{Y^{n}}, n \geq 1$, where $Y^{n} \subset \mathcal{N}$ and

$$
Y^{n}(t)=K(t, 0) x_{0}(0)+\int_{0}^{t} K(t, x) \overline{\mathrm{co}} F\left(s, X_{s}^{n-1}\right) d s .
$$

First of all, let us note that all $X^{n}, n \geq 1$ are nonempty since $\Sigma_{x_{0}} \subset X^{n}$ for all $n \geq 0$.

Let us now show that all sets $X^{n}, n \geq 1$ are equicontinuous. In fact, let $y \in X^{n}, n \geq 1$ and $t^{\prime}, t^{\prime \prime} \in[0, T], t^{\prime}<t^{\prime \prime}$. Then

$$
\begin{aligned}
\left\|y\left(t^{\prime \prime}\right)-y\left(t^{\prime}\right)\right\| \leq & \left\|K\left(t^{\prime \prime}, 0\right) x_{0}(0)-K\left(t^{\prime}, 0\right) x_{0}(0)\right\| \\
& +\int_{0}^{t^{\prime}}\left\|K\left(t^{\prime \prime}, s\right)-K\left(t^{\prime}, s\right)\right\| \cdot\|f(s)\| d s+\int_{t^{\prime}}^{t^{\prime \prime}}\left\|K\left(t^{\prime \prime}, s\right)\right\| \cdot\|f(s)\| d s
\end{aligned}
$$

where $f \in S_{\overline{\mathrm{co}} F\left(\cdot, X_{(\cdot)}^{n-1}\right)}$. Hence

$$
\begin{aligned}
\left\|y\left(t^{\prime \prime}\right)-y\left(t^{\prime}\right)\right\| \leq & \left\|\left(K\left(t^{\prime \prime}, 0\right)-K\left(t^{\prime}, 0\right)\right) x_{0}(0)\right\| \\
& +\int_{0}^{t^{\prime}}\left\|K\left(t^{\prime \prime}, s\right)-K\left(t^{\prime}, s\right)\right\| \gamma(s) d s+r \int_{t^{\prime}}^{t^{\prime \prime}} \gamma(s) d s
\end{aligned}
$$

giving the desired equicontinuity.

Now let us consider on the set $N$ the following measure of noncompactness

$$
\psi(\Omega)=\sup _{t \in[0, T]}\left[\varphi\left(\Omega_{t}\right) \exp \left(-R \int_{0}^{t} k(s) d s\right)\right]
$$

where $\varphi$ is a measure of noncompactness in $\mathcal{C}$ defined in Lemma $1.2, R>r$ is an arbitrary number and $k$ is the function from the estimation (F4). It is easy to see that the measure $\psi$ is monotone and nonsingular. From the proved equicontinuity property it follows that $\psi$ is regular on subsets $X^{n}, n \geq 1$.

Using Lemma 1.1 and the condition (F4) we have the following estimation

$$
\begin{aligned}
\chi\left(K(t, s) \overline{\mathrm{co}} F\left(s, X_{s}^{n-1}\right)\right) & \leq\|K(t, s)\| \chi\left(\overline{\mathrm{co}} F\left(s, X_{s}^{n-1}\right)\right) \\
& \leq r \chi\left(F\left(s, X_{s}^{n-1}\right)\right) \leq r k(s) \varphi\left(X_{s}^{n-1}\right) \\
& \leq r k(s) \psi\left(X^{n-1}\right) \exp \left(R \int_{0}^{s} k(\theta) d \theta\right)
\end{aligned}
$$

for all $s \in[0, t]$.

By virtue of the condition (F3') the multifunction

$$
s \multimap K(t, s) F\left(s, X_{s}^{n-1}\right), \quad s \in[0, T]
$$

is integrably bounded and hence, using Lemma 1.4, we have for any $t \in[0, T]$

$$
\begin{aligned}
\chi\left(Y^{n}(t)\right) & =\chi\left(K(t, 0) z(0)+\int_{0}^{t} K(t, s) \overline{\operatorname{co}} F\left(s, X_{s}^{n-1}\right) d s\right) \\
& =\chi\left(\int_{0}^{t} K(t, s) \overline{\operatorname{co}} F\left(s, X_{s}^{n-1}\right) d s\right) \\
& \leq r \psi\left(X^{n-1}\right) \int_{o}^{t} k(s) \exp \left(R \int_{0}^{s} k(\theta) d \theta\right) d s
\end{aligned}
$$




$$
\leq(r / R) \psi\left(X^{n-1}\right) \exp \left(R \int_{0}^{t} k(s) d s\right) .
$$

Therefore

$$
\chi\left(Y^{n}(t)\right) \exp \left(-R \int_{0}^{t} k(s) d s\right) \leq(r / R) \psi\left(X^{n-1}\right)
$$

and

$$
\psi\left(Y^{n}\right) \leq(r / R) \psi\left(X^{n-1}\right)
$$

Finally, we have $\psi\left(X^{n}\right) \leq(r / R) \psi\left(X^{n-1}\right), n \geq 1$ and therefore $\psi\left(X^{n}\right) \underset{n \rightarrow \infty}{\longrightarrow} 0$.

Applying Lemma 1.3 we obtain a compact set $X=\bigcap_{n \geq 1} X^{n}$ which has the desired properties.

Now consider a metric projection $P:[0, T] \times \mathcal{C} \multimap \mathcal{C}$,

$$
P(t, c)=\left\{e \in X_{t} \mid\|c-e\|=\operatorname{dist}\left(c, X_{t}\right)\right\}
$$

and a multimap $\widetilde{F}:[0, T] \times \mathcal{C} \rightarrow K v(E)$, defined by $\widetilde{F}(t, c)=\overline{\operatorname{co}} F(t, P(t, c))$. It is clear that $\widetilde{F}$ satisfies the properties (F3') and (F4). Following the arguments of [32] one can show that $\widetilde{F}$ satisfies also $(\mathrm{F} 1)$ and $(\mathrm{F} 2)$. Therefore the set $\Sigma_{x_{0}} \widetilde{F}_{\text {f }}$ all mild solutions of the problem

$$
\begin{aligned}
x^{\prime}(t) & \in A(t) x(t)+\widetilde{F}\left(t, x_{t}\right), & & t \in[0, T] ; \\
x(t) & =x_{0}(t), & & t \in[-\tau, 0]
\end{aligned}
$$

is nonempty. Moreover, the following statement is valid.

LEMMA 2.2. $\Sigma_{x_{0}}^{\widetilde{F}}=\Sigma_{x_{0}}^{F}$.

Proof. In fact, let $x \in \Sigma_{x_{0}}^{\widetilde{F}}$. Then

$$
\begin{aligned}
x(t) & \in K(t, 0) x_{0}(0)+\int_{0}^{t} K(t, s) \widetilde{F}(s, x(s)) d s \\
& =K(t, 0) x_{0}(0)+\int_{0}^{t} K(t, s) \overline{\operatorname{co}} F\left(s, P\left(s, x_{s}\right)\right) d s \\
& \subset K(t, 0) x_{0}(0)+\int_{0}^{t} K(t, s) \overline{\operatorname{co}} F\left(s, X_{s}\right) d s \subset X(t),
\end{aligned}
$$

i.e. $x \in X$ and $P\left(t, x_{t}\right)=\left\{x_{t}\right\}$. Then $x(t)=K(t, 0) x_{0}(0)+\int_{0}^{t} K(t, s) f(s) d s$, where $f \in S_{\widetilde{F}\left(\cdot, x_{(\cdot))}\right.}^{1}=S_{F\left(\cdot, x_{(\cdot)}\right)}^{1}$, hence $x \in \Sigma_{x_{0}}^{F}$.

The inclusion $\Sigma_{x_{0}}^{F} \subset \Sigma_{x_{0}}^{\widetilde{F}}$ easily follows from the observation that $\Sigma_{x_{0}}^{F} \subset X$.

Theorem 1.1 of [31] yields the following approximation result.

LEMma 2.3. There exists a sequence of multimaps $\left\{F_{n}\right\}_{n=1}^{\infty}, F_{n}:[0, T] \times \mathcal{C} \rightarrow K v(E)$ such that:

(i) each $F_{n}, n \geq 1$ satisfies the property (F1);

(ii) each multimap $F_{n}(t, \cdot): \mathcal{C} \rightarrow K v(E)$ is continuous for a.e. $t \in[0, T]$; 
(iii) $\widetilde{F}(t, c) \subset \ldots \subset F_{n+1}(t, c) \subset F_{n}(t, c) \subset \overline{\mathrm{co}} F\left(t, X_{t}\right), n \geq 1$;

(iv) $\widetilde{F}(t, c)=\bigcap_{n \geq 1} F_{n}(t, c)$

(v) for each $n \geq 1$ there exists a selection $g_{n}(t, c) \in F_{n}(t, c)$ which is measurable in $t$ for every $c \in \mathcal{C}$ and locally Lipschitz in $c$ in the sense that each $c_{0} \in \mathcal{C}$ has a neighborhood $U\left(c_{0}\right)$ and constant $k\left(c_{0}\right) \geq 1$ such that

$$
\left\|g_{n}\left(t, c_{1}\right)-g_{n}\left(t, c_{2}\right)\right\| \leq k\left(c_{0}\right) \gamma(t)\left\|c_{1}-c_{2}\right\| \quad \text { for a.e. } t \in[0, T]
$$

for $c_{1}, c_{2} \in U\left(c_{0}\right)$ where $\gamma(t)$ is the function from the estimation (F3').

Proof of Theorem 2.2. From (i)-(iii) of Lemma 2.3 it follows that each $F_{n}$ satisfies the conditions $(\mathrm{F} 1)-(\mathrm{F} 4)$ and hence each set $\Sigma_{x_{0}}^{F_{n}}, n \geq 1$ is nonempty and compact.

Let us fix $n \geq 1$ and demonstrate that the set $\Sigma_{x_{0}}^{F_{n}}$ is contractible. In fact, let $x_{n} \in \Sigma_{x_{0}}^{F_{n}}$ and for any $\tau \in[0,1)$ the function $z_{n}^{\tau}$ be a unique solution of the integral equation

$$
z_{n}(t)=K(t, \tau T) x_{n}(\tau T)+\int_{\tau T}^{t} K(t, s) g_{n}\left(s, z_{n}(s)\right) d s
$$

The functions

$$
w_{n}^{\tau}(t)= \begin{cases}x_{n}(t) & 0 \leq t \leq \tau T \\ z_{n}^{\tau}(t) & \tau T \leq t \leq T\end{cases}
$$

belong to $\Sigma_{x_{0}}^{F_{n}}$. Define the deformation $h:[0,1] \times \Sigma_{x_{0}}^{F_{n}} \rightarrow \Sigma_{x_{0}}^{F_{n}}$ by the formula

$$
h\left(\tau, x_{n}\right)= \begin{cases}w_{n}^{\tau} & \text { for } 0 \leq \tau<1, \\ x_{n} & \text { for } \tau=1 .\end{cases}
$$

Since the evolution operator $K(t, s)$ is strongly continuous and the function $g_{n}$ is locally Lipschitz, it is easy to see that the solutions of the problem (3) depend continuously on $\left(\tau, x_{n}\right)$, therefore the deformation $h$ is continuous. But $h(0, \cdot)=z_{n}^{0}$ and $h(1, \cdot)$ is the identity, hence $\Sigma_{x_{0}}^{F_{n}}$ is contractible.

Now let us show that

$$
\Sigma_{x_{0}}^{\widetilde{F}}=\bigcap_{n \geq 1} \Sigma_{x_{0}}^{F_{n}}
$$

It is clear that

$$
\Sigma_{x_{0}}^{\widetilde{F}} \subset \bigcap_{n \geq 1} \Sigma_{x_{0}}^{F_{n}}
$$

Let $x \in \bigcap_{n \geq 1} \Sigma_{x_{0}}^{F_{n}}$, then for each $n \geq 1$ we have

$$
x(t)=K(t, 0) x_{0}(0)+\int_{0}^{t} K(t, s) f_{n}(s) d s
$$

where $f_{n} \in S_{F_{n}\left(\cdot, x_{(\cdot)}\right)}^{1}$. But from (iii) of Lemma 2.3 it follows that $\bigcup_{n \geq 1} f_{n}(t)$ is relatively compact for a.e. $t \in[0, T]$ and, hence, we may assume, without loss of generality that

$$
f_{n} \underset{w}{\longrightarrow} f \in L^{1}([0, T] ; E)
$$

(see $[10])$.

According to the Mazur's Lemma (see, e.g. [12]) there exists a double sequence $\left\{\lambda_{i k}\right\}_{i=1}^{\infty} \underset{k=1}{\infty}$ such that: 
(1) $\sum_{k=i}^{\infty} \lambda_{i k}=1$ for all $i=1,2, \ldots$;

(2) $\lambda_{i k}=0$ for all $k \geq k_{0}(i)$;

(3) the sequence $\left\{\widetilde{f}_{i}\right\}_{i=1}^{\infty}, \widetilde{f}_{i}(t)=\sum_{k=1}^{\infty} \lambda_{i k} f_{k}(t)$ converges to $f$ with respect to the norm of the space $L^{1}([0, T] ; E)$. Passing to a subsequence, if necessary, we may set that $\widetilde{f}_{i}$ converges to $f$ a.e. on $[0, T]$.

But it is clear that $\widetilde{f}_{i}(t) \in F_{i}\left(t, x_{t}\right)$ for a.e. $t \in[0, T]$ and hence from (iv) of Lemma 2.3 it follows that $f \in S_{\widetilde{F}\left(\cdot, x_{(\cdot)}\right)}^{1}$.

For each $t \in[0, T]$ the map $q \rightarrow \int_{0}^{t} K(t, s) q(s) d s$ is a continuous linear operator from $L^{1}([0, T] ; E)$ into $E$. It remains continuous provided these spaces are endowed with weak topologies. Therefore for each $t \in[0, T]$ the sequence

$$
\left\{K(t, 0) x_{0}(0)+\int_{0}^{t} K(t, s) f_{n}(s) d s\right\}
$$

converges weakly to $K(t, 0) x_{0}(0)+\int_{0}^{t} K(t, s) f(s) d s$. But since each number of this sequence is equal to $x$ we have $x \in \Sigma_{x_{0}}^{\widetilde{F}}$ and hence

$$
\Sigma_{x_{0}}^{\widetilde{F}}=\bigcap_{n \geq 1} \Sigma_{x_{0}}^{F_{n}} .
$$

Therefore $\Sigma_{x_{0}}^{F}=\Sigma_{x_{0}}^{\widetilde{F}}$ is an $R_{\delta}$-set.

3. Example. The solutions set for the problem of a controlled heat transfer in an isotropic rigid body. Let $G \subset \mathbb{R}^{3}$ be an open domain with a smooth boundary $\partial G$. The function $z(t, y), z \in C\left([-\tau, T] ; L^{2}(G)\right)$ describes the temperature in the point $y \in G$ at the moment $t \in[-\tau, T]$.

Let there be $m$ sources of heat in the region $G$ whose properties depend on the temperature and whose densities are characterized by the functions $\Delta_{i}(y, z), \Delta_{i}: G \times$ $\mathbb{R} \rightarrow \mathbb{R}, i=1, \ldots, m$. The intensity of the sources can be regulated by the controls $u_{i}:[0, T] \rightarrow \mathbb{R}, i=1, \ldots, m$. It is supposed that $u_{i}$ are measurable functions satisfying the following feedback condition

$$
\left(u_{1}(t), \ldots, u_{m}(t)\right) \in W(t, z(t-w(t), \cdot))
$$

where $w:[0, T] \rightarrow[0, \tau]$ is a continuous function, $W:[0, T] \times L^{2}(G) \rightarrow K v\left(\mathbb{R}^{m}\right)$ is a bounded multimap measurable in the first argument and continuous in the second one.

Let $p: G \rightarrow \mathbb{R}_{+}$be the coefficient of heat conductivity. Then the controlled process of heat transfer in the domain $G$ can be described by the following relations (see [3], [22]):

$$
\begin{aligned}
\frac{\partial z(t, y)}{\partial t} & =\sum_{k=1}^{3} \frac{\partial}{\partial y_{k}} p(y) \frac{\partial}{\partial y_{k}} z(t, y)+\sum_{i=1}^{m} u_{i}(t) \Delta_{i}(y, z(t, y)) ; \\
\left.z(t, \cdot)\right|_{\partial G} & =0, t \in[-\tau, T] ; \\
z(t, \cdot) & =x_{0}(t), t \in[-\tau, 0] ; x_{0} \in C\left([-\tau, 0] ; L^{2}(G)\right) .
\end{aligned}
$$


Assume that the functions $\Delta_{i}$ satisfy for every $i=1, \ldots, m$ the following conditions:

$(\Delta 1) \Delta_{i}(\cdot, z): G \rightarrow \mathbb{R}$ is measurable for all $z \in \mathbb{R}$

$(\Delta 2)\left|\Delta_{i}(y, z)\right| \leq \alpha_{i}(y)$ for all $z \in \mathbb{R}$ where $\alpha_{i} \in L_{+}^{2}(G)$;

$(\Delta 3)\left|\Delta_{i}\left(y, z_{1}\right)-\Delta_{i}\left(y, z_{2}\right)\right| \leq k_{i}\left|z_{1}-z_{2}\right|$ for all $z_{i}, z_{2} \in \mathbb{R}$ where $k_{i}$ doesn't depend on $y \in G$.

By standard methods (see, for example, [19], [4]) the problem (4)-(7) can be reduced to a delay differential inclusion in a Banach space $L^{2}(G)$

$$
x^{\prime}(t) \in A x(t)+F(t, x(t), x(t-w(t)))
$$

where $x(t)$ is generated by the function $z(t, \cdot), A$ is a differential operator and

$$
\begin{aligned}
F(t, x(t), x(t-w(t)))= & \left\{v \in L^{2}(G): v(y)=\sum_{i=1} w_{i}(t) \Delta_{i}(y, x(t)(y)),\right. \\
& \left.\left(w_{1}(t), \ldots, w_{m}(t)\right) \in W(t, x(t-w)) \text { are measurable }\right\} .
\end{aligned}
$$

It was shown in [22] that under the above conditions the multimap $F$ satisfies the assumptions (F1)-(F4) and, hence, by Theorem 2.2, we conclude that the set of all mild solutions $z(t, y)$ of the problem (4)-(7) form an $R_{\delta}$-set in the space $C\left([-\tau, T] ; L^{2}(G)\right)$.

\section{References}

[1] R. R. Akhmerov, M. I. Kamenskiŭ, A. S. Potapov, A. E. Rodkina and B. N. Sadovskiı̌, Measures of Noncompactness and Condensing Operators, Birkhäuser Verlag, Basel-Boston-Berlin, 1992.

[2] G. Anichini, G. Conti and P. Zecca, Using solution sets for solving boundary value problems for ordinary differential equations, Nonlinear Anal., Theory, Meth. and Appl. 17 No. 5 (1991), 465-472.

[3] G. Anichini and P. Zecca, Multivalued differential equations in Banach spaces, an application to control theory, J. Optim. Theory and Appl. 21 No. 4 (1977), 477-486.

[4] Yu. G. Borisovich, B. D. Gelman, A. D. Myshkis and V. V. Obukhovskiǔ, Introduction to the Theory of Multivalued Maps, Voronezh Univ. Press, Voronezh, 1986 (in Russian).

[5] C. Castaing and M. Valadier, Convex Analysis and Measurable Multifunctions, Lect. Notes in Math. 580, Springer, Berlin, 1977.

[6] S. N. Chow and J. D. Schuur, Fundamental theory of contingent differential equations in Banach spaces, Trans. Amer. Math. Soc. 179 (1973), 133-144.

[7] L. J. Davy, Properties of the solution set of a generalized differential equation, Bull. Australian Math. Soc. 6 (1972), 379-398.

[8] F. DeBlasi, Existence and stability of solutions for autonomous multivalued differential equations in Banach spaces, Rend. Acad. Naz. Lincei, Serie VII, 60 (1976), 767-774.

[9] F. DeBlasi and J. Myjak, On the solution sets for differential inclusions, Bull. Pol. Acad. Sci. 33 (1985), 17-23.

[10] J. Diestel, Remarks on weak compactness in $L_{1}\left(\mu_{1}, X\right)$, Glasgow Math. J. 18, No. 1 (1977), 87-91. 
[11] G. Dragoni, J. Macki, P. Nistri and P. Zecca, Solution sets of differential equations in abstract spaces, Pitman Res. Notes in Math., Longman, to appear.

[12] I. Ekeland and R. Temam, Convex Analysis and Variational Problems, North-Holland, Amsterdam, 1976.

[13] C. Himmelberg and F. Van Vleck, A note on the solution sets for differential inclusions, Rocky Mountain J. Math. 12 (1982), 621-625.

[14] D. M. Hyman, On decreasing sequences of compact absolute retracts, Fund. Math. 64 (1969), 91-97.

[15] M. I. Kamenskiŭ, P. Nistri, V. V. Obukhovskiŭ and P. Zecca, Optimal feedback control for a semilinear evolution equation, J. Optim. Theory and Appl. 82 No. 3 (1994), 503-517.

[16] M. Kisiliewicz, Multivalued differential equations in separable Banach spaces, J. Optim. Th. Appl. 37 (1982), 231-249.

[17] S. G. Krein, Linear Differential Equations in Banach Spaces, Amer. Math. Soc., Providence, 1971.

[18] J. M. Lasry and R. Robert, Acyclicité de l'ensemble des solutions de certaines équations fonctionnelles, C. R. Acad. Sci. Paris 282 (1976), 1283-1286.

[19] R. Martin, Nonlinear Operators and Differential Equations in Banach Spaces, Wiley, New York, 1976.

[20] A. M. Muhsinov, On differential inclusions in Banach spaces, Soviet Math. Dokl. 15 (1974), 1122-1125.

[21] P. Nistri, V. V. Obukhovskiı̆ and P. Zecca, On the solvability of systems of inclusions involving noncompact operators, Trans. Amer. Math. Soc. 342, No. 2 (1994), 543-562.

[22] V. V. Obukhovskiŭ, Semilinear functional differential inclusions in a Banach space and controlled parabolic systems, Soviet J. Automat. Inform. Sci. 24, No. 3 (1991), 71-79 (1992).

[23] N. S. Papageorgiou, On multivalued evolution equations and differential inclusions in Banach spaces, Comment. Math. Univ. Sancti Pauli 36, No. 1 (1987), 21-39.

[24] N. S. Papageorgiou, On the solution set of differential inclusions in Banach space, Appl. Anal. 25 (1987), 319-329.

[25] N. H. Paovel and J. Vrabie, On the solution set of differential inclusions with state constraints, Appl. Anal. 31 (1989), 279-289.

[26] R. M. Sentis, Convergence de solutions d'équations différentielles multivoques, C.R. Acad. Sci. Paris, Série A, 278 (1974), 1623-1626.

[27] A. A. Tolstonogov, On differential inclusions in Banach spaces and continuous selectors, Dokl. Akad. Nauk SSSR 244 (1979), 1088-1092.

[28] A. A. Tolstonogov, On properties of solutions of differential inclusions in Banach spaces, Dokl. Akad. Nauk SSSR 248 (1979), 42-46.

[29] A. A. Tolstonogov, On the structure of the solution set for differential inclusions in a Banach space, Math. Sbornik 46 (1983), 1-15.

[30] A. A. Tolstonogov, Differential Inclusions in a Banach Space, Nauka, Novosibirsk, 1986 (in Russian).

[31] A. A. Tolstonogov and Ya. I. Umanskiŭ, On solutions of evolution inclusions II, Sibirsk. Mat. Zh. 33, No. 4 (1992), 163-174 (in Russian).

[32] Ya. I. Umanskiŭ, On a property of solutions set of differential inclusions in a Banach space, Differ. Uravneniya 28, No. 8 (1992), 1346-1351 (in Russian). 